\title{
Parámetros hematológicos en caballos peruanos de paso en la provincia de Lambayeque
}

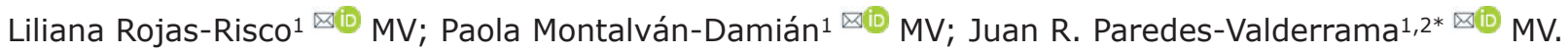

\begin{abstract}
${ }_{1}^{1}$ Universidad Nacional Pedro Ruíz Gallo, Facultad de Medicina Veterinaria, Laboratorio de Patología Clínica. Lambayeque, Perú. ¿Universidad Privada Antenor Orrego, Escuela de Posgrado. Trujillo, Perú.

* Correspondencia: juanparedes1912@hotmail.com
\end{abstract}

Recibido: Marzo 2020; Aceptado: Junio 2020; Publicado: Agosto 2020.

\section{RESUMEN}

Objetivo. Determinar los parámetros hematológicos según el estado reproductivo en caballos peruanos de Paso en la provincia de Lambayeque. Materiales y métodos. Se realizó la investigación con 60 caballos adultos (>4 años), clínicamente sanos y sin tratamiento farmacológico previo; según su estado reproductivo se formaron cuatro grupos de 15 ejemplares cada uno: macho castrado, macho entero, yegua vacía y yegua gestante. Primero se realizó el examen clínico general para confirmar su buen estado de salud, luego se extrajo sangre de la vena yugular a todos los caballos. Las muestras se procesaron por análisis hematológicos y se determinaron las variables de la serie roja (hemoglobina, hematocrito, glóbulos rojos, volumen corpuscular medio [VCM], hemoglobina corpuscular media [HCM] y concentración de hemoglobina corpuscular media [CHCM]). Igualmente, la serie blanca (leucocitos, neutrófilos segmentados y abastonados, basófilos, monocitos, linfocitos, eosinófilos) y serie trombocítica fueron procesados y determinados. Se utilizaron métodos manuales, fórmulas matemáticas y método Drabkin en el laboratorio de patología clínica. Se aplicó la prueba estadística de Kruskal Wallis y se obtuvo la media, desviación estándar y rangos de las variables. Resultados. No se encontraron diferencias estadísticamente significativas en la serie blanca, roja y trombocítica de acuerdo con el sexo o estado reproductivo, a excepción del número de los basófilos que fue mayor en machos enteros. Conclusiones. Los parámetros hematológicos según el estado reproductivo del caballo peruano de paso se encuentran dentro de los rangos establecidos por la literatura especializada. Sin embargo, el número los linfocitos que fue mucho menor.

Palabras clave: Caballos; estado reproductivo; parámetros hematológicos (Fuente: DECs).

\section{ABSTRACT}

Objective. To determine the haematological parameters according to the reproductive status of Peruvian Paso horses in the Province of Lambayeque. Materials and methods. The research was conducted on 60 clinically healthy adult horses ( $>4$ years), without previous drug treatment. Four groups of 15 horses were formed: gelding, entire male, empty mare and pregnant mare. First, a general clinical examination was performed to confirm their good health. Blood was drawn from 
the jugular vein of all the horses. The samples were processed by haematological analysis and the parameters of the red series (haemoglobin, haematocrit, red blood cells, mean corpuscular volume $[\mathrm{MCV}]$, mean corpuscular haemoglobin $[\mathrm{MCH}]$ and the mean corpuscular haemoglobin concentration $[\mathrm{MCHC}]$ were determined. Likewise, the white series (leukocytes, neutrophils segmented and stacked, basophils, monocytes, lymphocytes, eosinophils) and the thrombocytic series were processed and the unit range determined. Manual methods, mathematical formulas and the Drabkin method were used in the clinical pathology laboratory. The Kruskal Wallis Statistical test was applied and consequently the mean, standard deviation and ranges of variables were obtained. Results. No significant statistical differences were found in the white, red and thrombocytic series according to sex or reproductive status, except the number of basophils was higher in the entire males. Conclusions. The haematological parameters according to the reproductive status of the Peruvian Step horse are within the normal ranges (as per the literature pertaining to this area). However, the number of lymphocytes was much lower.

Keywords: Horses; reproductive status; haematological parameters (Source: DECS).

\section{INTRODUCCIÓN}

El caballo desde tiempos inmemorables se utiliza por parte del hombre para la ejecución de muchas tareas, aunque en las últimas décadas demostró ser útil para contrarrestar discapacidades psicomotoras y como atenuante en niños con parálisis cerebral $(1,2)$.

Los parámetros hematológicos, enzimáticos o bioquímicos son muy variables en los caballos alrededor del mundo; ya sea por la edad, raza, sexo, tipo de trabajo, temperatura o algunas patologías. En el caso de caballos de resistencia con alto rendimiento, los niveles de potasio y lactato son mayores comparados con los de bajo rendimiento (3). De igual manera que los valores de hemoglobina, hierro y cobre son más bajos en los caballos que padecen "pica" (4). En equinos adultos mayores de 15 años, las concentraciones de proteínas plasmáticas, leucocitos, neutrófilos, hemoglobina y hematocrito son mayores en comparación con aquellos de menor edad y expuestos al ejercicio constante $(5,6)$, pudiendo presentar algunas hembras cambios en su perfil hematológico después del ejercicio (7). Asimismo, el ambiente pudiera influir en los parámetros bioquímicos del suero, sobre todo en analitos como AST (Aspartato aminotransferasa) y CK (creatinina cinasa); en caballos criados en el trópico (8).

Los valores hematológicos son una importante herramienta para calificar el estado físico-general de los caballos y establecer un posible pronóstico ante una determinada patología $(9,10)$. Pero hasta la hecha, no existen evidencias de estos valores para la evaluación del caballo Peruano de Paso según su estado reproductivo; que se aplique como referencia en la práctica clínica. Ello ha sido una de las grandes barreras a las que han tenido que enfrentarse aquellos médicos veterinarios dedicados a la clínica médica equina.

El presente estudio tuvo como objetivo; determinar los parámetros hematológicos del Caballo Peruano de Paso, debido a la escasa información respecto a los parámetros hematológicos en los diferentes estados reproductivos.

\section{MATERIALES Y MÉTODOS}

Ubicación del estudio. Este estudio de tipo transversal observacional fue realizado entre los meses de enero a febrero del 2019; en diferentes criaderos pertenecientes a la Asociación de Criadores y Propietarios de Caballos Peruanos de Paso de la Provincia de Lambayeque - Perú. La zona se ubica al norte del país y cuenta con una temperatura promedio de $22.5^{\circ} \mathrm{C}$, humedad relativa del $48 \%$ y 18 m.s.n.m. ubicándose entre las coordenadas $6^{\circ} 42^{\prime} 17^{\prime \prime S} 79^{\circ} 54^{\prime} 25^{\prime \prime}$ O.

Selección de los animales. Se seleccionaron 60 equinos con buena condición corporal, mayores de 4 años (con una media de $8.8 \pm 3.9$ años) y con libre acceso a agua y con igual proporción entre machos y hembras, discriminados por su estado reproductivo en cuatro grupos de investigación de 15 ejemplares cada uno: hembra vacía (G1), hembra gestante (G2); macho castrado (G3) y macho entero (G4). El régimen alimentario estuvo constituido por alfalfa (Medicago sativa), sorgo forrajero (Sorghum sp.) y maralfalfa (Pennisetum sp.), principalmente; en una proporción del $2 \%$ de su 
peso corporal, conjuntamente con balanceado (Omolin ${ }^{\circledR}$ de Purina).

Manejo de los animales previo a la toma de muestra y criterios de exclusión. Los ejemplares estuvieron en ayuno previo a la toma de las muestras sanguíneas y con un reposo de 30 minutos, se les practicó un examen clínico general con el fin de corroborar su correcto estado de salud y edad promedio mediante cronometría dentaria. No se incluyeron en el estudio caballos con tratamiento farmacológico dentro de los tres meses previos a la toma de muestras sanguíneas o con presencia de alguna patología.

Examen clínico. Se realizaron los métodos de exploración clínica, que se basaba en la inspección, palpación, percusión y auscultación. Los animales se observaron de frente, por ambos lados y desde atrás para tener una mejor valoración de la apariencia general del cuerpo, postura, temperamento, signos de dolor, debilidades, estado de la piel y pelaje. Asimismo, se analizaron la frecuencia y profundidad de la respiración, existencia de heridas, inflamaciones, asimetrías en el desarrollo muscular y posibles exudados presentes en los orificios naturales. Se prestó atención en las mucosas orales y oculares, en búsqueda de anormalidades en el color, textura y conformación dentaria. Se palparon las extremidades anteriores en las regiones preescapular y pectoral, en búsqueda de la existencia de zonas con calor, dolor o inflamación, así como anomalías de conformación. Se calcularon la frecuencia cardiaca (FC) y respiratoria (FR), el pulso digital y la temperatura corporal. Se tomaron como referencia los rangos fisiológicos normales para esta especie: $F C=28-40$ latidos/min, $F R=8-20$ respiraciones/min y Temperatura corporal= $37.5-38.5^{\circ} \mathrm{C}$. Se realizó el examen del abdomen, mediante auscultación de la fosa paralumbar y las regiones ventrales del flanco para evaluar los movimientos peristálticos y sonidos provenientes de la válvula ileocecal. Este procedimiento también permitió evaluar las características de los ruidos intestinales (normales, aumentados, disminuidos o ausentes). En la región ventral derecha se auscultó el colon ventral derecho; en la fosa paralumbar izquierda, el intestino delgado y en la región ventral izquierda, el colon ventral izquierdo. En el caso de yeguas se examinó la vulva (conformación, tono y color de la mucosa) en búsqueda de posibles descargas anormales. En contraste se palparon en lo machos el prepucio, pene y testículos para verificar la existencia o no de neoplasias (11).
Toma de muestras de sangre. Previo a la toma de muestra se realizó la antisepsia de la zona del surco yugular con alcohol al 90\%. De cada animal se tomó $3 \mathrm{ml}$ de sangre, con ayuda de un Holder y una aguja $\left(21 \times 1^{1 / 2^{\prime}}\right)$, en un tubo de colecta con anticoagulante EDTA (ácido etilendiaminotetraacético). Se homogenizó y rotuló la muestra indicando la edad, sexo y procedencia del animal. Las muestras se transportaron refrigeradas $\left(2-5^{\circ} \mathrm{C}\right)$ en recipiente hermético (Cooler) con gel refrigerante hasta el Laboratorio de Patología Clínica de la Facultad de Medicina Veterinaria de la Universidad Nacional Pedro Ruiz Gallo. El tiempo trascurrido entre la obtención de muestras sanguíneas y análisis de laboratorio fue de 45 minutos en promedio.

Análisis de muestras de sangre. Para la determinación del hematocrito $(\mathrm{Ht})$ se utilizó tubos capilares heparinizados, que fueron selladas en un extremo con plastilina; que luego se centrifugaron durante tres minutos a $30000 \mathrm{rpm}$ en una microcentrífuga. Los resultados se interpretaron de acuerdo a la tabla de hematocrito del 0-100 expresándose en porcentaje (12).

La hemoglobina $(\mathrm{Hb})$ se calculó a través de la homogenización de $2.5 \mathrm{ml}$ de reactivo Drabkin más $10 \mu \mathrm{L}$ de sangre que fueron dejadas en reposo por un lapso de tres minutos. Los tubos de ensayo fueron leídos en el fotocolorímetro a una longitud de onda de $550 \mathrm{~nm}$, utilizando también un tubo blanco con la solución Drabkin que sirvió como calibrador (12)

El conteo de Eritrocitos Totales (ET) fue por medio de la cámara de Neubauer. Para ello se diluyó 1 $\mathrm{ml}$ de reactivo Gower con $5 \mu \mathrm{L}$ de sangre con anticoagulante. Invirtiendo cuidadosamente los tubos varias veces con el fin de lograr una mejor mezcla y dejándolo reposar 3 minutos. Se introdujo entre la cámara de Neubauer y el cubreobjetos $10 \mu \mathrm{L}$ de la solución para que fluyera por medio de la capilaridad para luego dejarla reposar otros 3 minutos. El conteo se realizó bajo microscopio óptico a 40X, empleándose la siguiente fórmula: células contadas x 10000 (12).

Las constantes corpusculares se dedujeron por medio de las siguientes fórmulas:

$\mathrm{VCM}=\mathrm{Ht} \times 10 / \mathrm{ET} ; \mathrm{HCM}=\mathrm{Hb} \times 10 / \mathrm{ET}$ y

$\mathrm{CMHC}=\mathrm{Hb} \times 100 / \mathrm{Ht}(12,13)$

Para contabilizar el Total Leucocitario (TL), se mezcló $0.96 \mathrm{ml}$ del reactivo de Turk y $50 \mu \mathrm{L}$ de 
sangre, dejando reposar 3 minutos. Luego se introdujo por capilaridad $10 \mu \mathrm{L}$ de la solución entre la cámara de Neubauer y cubreobjetos, reposando otros tres minutos para su posterior conteo. Se siguió la siguiente fórmula $T L=$ células contadas x 50 (12).

El conteo diferencial de leucocitos se realizó en frotis de sangre con la tinción de Wright. Se contaron 100 células en total y se expresaron las cantidades observadas en porcentaje. Se agruparon los precursores mieloide y linfoide para facilitar la separación en la fórmula leucocitaria y poder establecer la comparación con los rangos fisiológicos de referencia $(12,13)$.

En el cálculo de las plaquetas totales (PT) se mezcló $2 \mathrm{ml}$ de oxalato de amonio y $20 \mu \mathrm{L}$ de sangre. Posteriormente, se colocó $10 \mu \mathrm{L}$ de la solución en la cámara de Neubauer, que se dejó incubar en una cámara húmeda por un lapso de 15 minutos. Las PT = células contadas X 1000 (12).

Análisis estadístico. Se aplicaron las pruebas de homogeneidad de varianza de Levene y Kruskal Wallis. Las posibles diferencias entre los grupos de estudio (estado reproductivo y sexo), se aplicó la prueba post hoc de Tukey. Considerándose estadísticamente diferente cada grupo cuando el valor $\mathrm{p}<0.05$. Se obtuvieron los estadígrafos de cada variable de la serie roja, blanca y trombocítica como la desviación estándar, media y rangos. Todo ello trabajado en el paquete estadístico SPSS Statistics v25.0.

Aspectos éticos. Todos los procedimientos llevados a cabo, durante la recolección de muestras y examen físico general; estuvieron dentro de los límites permitidos en el reglamento del Comité Institucional de Ética en Investigación con Animales y Biodiversidad de la Universidad Científica del Sur, aprobado en el 2017. En este estudio se salvaguardó la integridad y salud de los animales evaluados.

\section{RESULTADOS}

El hematocrito, hemoglobina y recuento eritrocitario fueron mayores; pero no estadísticamente significativos en machos enteros como se muestra en la tabla 1. Además, no se encontraron diferencias significativas en la serie roja en relación al estado reproductivo de los caballos.

En los valores de la serie blanca (Tabla 2), los valores de leucocitos y neutrófilos segmentados fueron menores en machos, pero mayores en neutrófilos abastonados. Además, existe significancia estadística entre los grupos; siendo favorecido los machos enteros; encontrándose mayor número de basófilos.

Tabla 1. Valores hematológicos de la serie roja entre grupos de investigación de equinos de la raza Caballo Peruano de Paso criados en Lambayeque.

\begin{tabular}{|c|c|c|c|c|c|c|}
\hline \multirow{2}{*}{ Serie roja } & \multicolumn{4}{|c|}{ Grupos } & \multirow{2}{*}{$\begin{array}{l}\text { Promedio } \\
\text { (max - min) }\end{array}$} & \multirow{2}{*}{ Valor $\mathbf{p} *$} \\
\hline & G1 $(n=15)$ & $G 2(n=15)$ & G3 $(n=15)$ & G4 $(n=15)$ & & \\
\hline GR $\left(X 10^{6} / \mu \mathrm{l}\right)$ & $7.3 \pm 0.8$ & $7.5 \pm 1.1$ & $7.7 \pm 0.9$ & $8.0 \pm 1.1$ & $\begin{array}{c}7.6 \pm 1.1 \\
(5.7-9.3)\end{array}$ & 0.26 \\
\hline$H b(g / d L)$ & $10.8 \pm 1.8$ & $11.4 \pm 2.3$ & $11.6 \pm 1.9$ & $12.4 \pm 2.2$ & $\begin{array}{c}11.6 \pm 2.1 \\
(8.0-15.4)\end{array}$ & 0.22 \\
\hline Ht (\%) & $35.3 \pm 4.1$ & $36.4 \pm 5.0$ & $36.9 \pm 3.8$ & $38.7 \pm 4.9$ & $\begin{array}{c}36.8 \pm 4.5 \\
(28.0-45.0)\end{array}$ & 0.24 \\
\hline HCM (pg) & $14.7 \pm 0.7$ & $15.1 \pm 1.1$ & $15.0 \pm 0.8$ & $15.4 \pm 1.0$ & $\begin{array}{l}15.0 \pm 0.9 \\
(13.4-6.6)\end{array}$ & 0.28 \\
\hline VCM (fL) & $48.6 \pm 0.6$ & $48.6 \pm 1.9$ & $48.0 \pm 2.2$ & $48.5 \pm 1.8$ & $\begin{array}{c}48.4 \pm 1.7 \\
(41.9-53.2)\end{array}$ & 0.77 \\
\hline CHCM $(g / d L)$ & $30.3 \pm 1.7$ & $31.1 \pm 2.3$ & $31.4 \pm 2.2$ & $31.8 \pm 2.2$ & $\begin{array}{c}31.2 \pm 2.1 \\
(27.7-35.3)\end{array}$ & 0.27 \\
\hline
\end{tabular}

G1=hembra vacía; G2=hembra gestante; G3=macho castrado; G4=macho entero; Mín=Mínimo; Máx=Máximo; GR= Glóbulos rojos; $\mathrm{Hb}=$ Hemoglobina; Hto= Hematocrito; $\mathrm{HCM}=$ Hemoglobina corpuscular media; VCM= Volumen corpuscular medio; $\mathrm{CHCM}=$ Concentración de hemoglobina corpuscular media; $* \mathrm{p}<0.05$ en relación con el estado reproductivo (Kruskal-Wallis) 
Tabla 2. Valores hematológicos de la serie blanca y trombocítica entre grupos de investigación de equinos de la raza Caballo Peruano de Paso criados en Lambayeque.

\begin{tabular}{|c|c|c|c|c|c|c|}
\hline \multirow{2}{*}{ Serie blanca } & \multicolumn{4}{|c|}{ Grupos } & \multirow{2}{*}{$\begin{array}{l}\text { Promedio } \\
(\max -\min )\end{array}$} & \multirow{2}{*}{ Valor $p^{*}$} \\
\hline & G1 $(n=15)$ & G2 $(n=15)$ & G3 $(n=15)$ & G4 $(n=15)$ & & \\
\hline Leu $(/ \mu \mathrm{L})$ & $10367 \pm 1529$ & $10470 \pm 2107$ & $9687 \pm 2166$ & $9667 \pm 1690$ & $\begin{array}{c}10048 \pm 1881 \\
(5700-13500)\end{array}$ & 0.51 \\
\hline $\operatorname{Seg}(/ \mu L)$ & $5037 \pm 1147$ & $5384 \pm 1834$ & $4970 \pm 1315$ & $4944 \pm 1255$ & $\begin{array}{c}5084 \pm 1388 \\
(2622-8190)\end{array}$ & 0.81 \\
\hline$A b(/ \mu L)$ & $39 \pm 50$ & $35 \pm 46$ & $68 \pm 45$ & $63 \pm 48$ & $\begin{array}{c}51 \pm 48 \\
(0-122)\end{array}$ & 0.14 \\
\hline $\operatorname{Eos}(/ \mu \mathrm{L})$ & $370 \pm 337$ & $444 \pm 278$ & $224 \pm 176$ & $388 \pm 293$ & $\begin{array}{c}357 \pm 282 \\
(0-976)\end{array}$ & 0.17 \\
\hline Bas $(/ \mu L)$ & $19 \pm 41$ & $51 \pm 73$ & $54 \pm 66$ & $82 \pm 57 a$ & $\begin{array}{c}52 \pm 63 \\
(0-239)\end{array}$ & 0.04 \\
\hline Lin $(/ \mu \mathrm{L})$ & $4795 \pm 1080$ & $4343 \pm 857$ & $4223 \pm 1356$ & $4086 \pm 1131$ & $\begin{array}{l}4362 \pm 1124 \\
(1650-6750)\end{array}$ & 0.35 \\
\hline Mon $(/ \mu L)$ & $144 \pm 111$ & $212 \pm 125$ & $148 \pm 140$ & $104 \pm 98$ & $\begin{array}{c}152 \pm 123 \\
(0-515)\end{array}$ & 0.11 \\
\hline Trb (miles $/ \mu \mathrm{L})$ & $167 \pm 25$ & $178 \pm 21$ & $173 \pm 21$ & $168 \pm 20$ & $\begin{array}{c}171 \pm 22 \\
(120-213)\end{array}$ & 0.46 \\
\hline
\end{tabular}

G1=hembra vacía; G2=hembra gestante; G3=macho castrado; G4=macho entero; Leu= Leucocitos; Seg= Neutrófilos segmentados; $A b=$ Neutrófilos abastonados; Eos= Eosinófilos; Bas= Basófilos; Lin= Linfocitos; Mon= Monocitos; Trb $=$ Trombocitos; $* p<0.05$ en relación con el estado reproductivo (Kruskal-Wallis).

a = diferente al resto del grupo según prueba post hoc de Tukey

\section{DISCUSIÓN}

Los exámenes hematológicos en la medicina veterinaria son las más solicitadas por los profesionales; debido a su rapidez y sencillez. Donde los resultados dan un panorama general del estado de salud del animal, la cual se complementa con el examen físico general e historia clínica. Los valores hematológicos son muy variables, pudiendo ser las causas de estas variaciones la nutrición, edad, raza entre otros. Sin embargo, los hallazgos encontrados en este estudio se ubican dentro de los parámetros que reporta la literatura especializada y laboratorios peruanos de prestigio que ejecutan análisis clínicos en esta especie. Reportándose que el número de linfocitos se encuentran por debajo del reportado por los laboratorios $(13,14)$.

El recuento total de eritrocitos encontrados está por debajo del hallado en caballos de paso del valle de Lurín con una media de $8.3 \times 10^{6} / \mu \mathrm{L}$ (15). El hematocrito, se halló en el límite inferior de caballos criollos en Brasil, con una media de $37.4 \%$ (16); pero por encima del encontrado en caballos frisones de América del Norte (34\%)

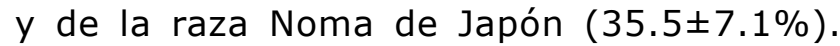
Además, este último presenta niveles elevados de eosinófilos $(383 \pm 529 / \mu \mathrm{l})$ y bajo en monocitos $(74 \pm 123 / \mu \mathrm{l})(17,18)$. Posiblemente los contrastes antes mencionados, se deba a diferencias en protocolos de sanidad, exposición a enfermedades, altitud de la región y alimentación. Siendo este último, probablemente el causante de cambios hematológicos en equinos (19).

Los picos de hemoglobina pueden diferir en un mismo animal, de acuerdo al tiempo de ejercicio que este realiza. Ello explica que los valores de hemoglobina del caballo peruano de paso son muy similares a los encontrados en caballos de raza Jeju mayores de 4 años en estado de reposo (20).

El nivel de basófilos en machos enteros $(82 \pm 57 / \mu \mathrm{l})$ fue el más elevado dentro de los grupos estudiados. Este valor se encuentra por encima de los encontrados en machos de 10 años de la raza Barb en Argelia; con una media de $53 \pm 47.51 / \mu \mathrm{l}$, donde también se evidenció que el número de basófilos fue mayor en machos (21). Aunque es común que la especie equina demuestre basofilia (12). 
Independientemente de que en este estudio no hubo diferencias significativas entre sexos, se corroboró que las hembras tienen mayor variabilidad en los parámetros hematológicos después del ejercicio (7), aunque en algunas razas (Lipizzano) esto puede tener un efecto contrario (22). Lo mismo pudiera ocurrir con el VCM, donde en ocasiones este es mayor en machos castrados mayores a 25 meses de edad (23), sin embargo, no se observaron diferencias entre los grupos estudiados.

El VCM es muy similar al de los caballos árabes sanos; pero se ubica por debajo de los niveles de HCM y CHCM con una media de $17.5 \mathrm{pg}$ y $36.64 \mathrm{gr} / \mathrm{dl}$, respectivamente. Además, los niveles de plaquetas fueron mucho más altas en comparación con la raza antes mencionada (24)

Asimismo, las hembras gestantes en periparto pueden tener valores hematológicos diferentes al resto del grupo; debido a deshidratación en el parto; causando hemoconcentración, estrés y desbalance energético $(25,26)$. En contraste, todo lo opuesto puede ocurrir en hembras vacías que poseen valores más altos en hemoglobina y recuento de eritrocitos, en comparación con yeguas gestantes andaluza y pura sangre (27). Por otro lado, las yeguas gestantes pesadas de tiro de Japón, clínicamente sanas; poseen valores linfocitarios menores $(2490 / \mu \mathrm{l})$ pero alto en número de neutrófilos segmentados $(5730 / \mu \mathrm{l})$, en comparación de la yegua gestante peruana de Paso (28). A diferencia de estos resultados, en este estudio no se observaron diferencias en las mismas variables.

En otro sentido, la toma de muestras del presente estudio se realizó en temporada de verano; época en la que algunos autores han comprobado la existencia de cambios hematológicos ocasionado por la temperatura ambiental, humedad relativa y precipitaciones donde la HCM es más alta en verano y los eosinófilos, en invierno (29). Por ello deben tenerse en cuenta los factores antes mencionados, para definir la extracción de muestras sanguíneas.
Como es de conocimiento, el caballo peruano de paso no es usado exclusivamente para el trabajo, sino más bien para exhibiciones y concursos. Durante el tiempo previo a dichos eventos, los caballos son sometidos a entrenamientos durante largos periodos; pudiendo denotar cambios hematológicos y bioquímicos en relación con aquellos que no son forzados a estas prácticas y ejercicios intensos $(30,31,32)$. Esto pudiera ser un factor condicionante, de cambios en los valores hematológicos en el Caballo Peruano de Paso.

Como se había señalado, no existen parámetros hematológicos de referencia en caballos peruanos de paso según su estado reproductivo. Los resultados obtenidos servirán como punto de partida para la elaboración de un tratamiento integral y a su vez, establecer un pronóstico sobre la salud general de los equinos de Lambayeque.

Se concluye que los parámetros hematológicos estudiados en los caballos peruanos de paso de la provincia Lambayeque, se mantienen en el rango fisiológico de referencia y son similares en los diferentes estados reproductivos

\section{Conflicto de intereses}

Los autores declaran no tener conflictos de intereses.

\section{Agradecimientos}

Un agradecimiento fraterno a los Médicos Veterinarios Elmer Plaza y Martín Laca, por las orientaciones durante el estudio. También a la Asociación de Criadores y Propietarios del Caballo Peruano de Paso-Lambayeque, por la confianza prestada y por abrirnos las puertas para poder realizar este trabajo. 


\section{REFERENCIAS}

1. Del Rosario-Montejo $O$, Molina-Rueda F, Muñoz-Lasa S, Alguacil-Diego IM. Effectiveness of equine therapy in children with psychomotor impairment. Neurology. 2015; 30(7):425-432. https://doi. org/10.1016/j.nrleng.2013.12.017

2. Drnach $M, O^{\prime} B$ rien $P A$, Kreger $A$. The effects of a 5-week therapeutic horseback riding program on gross motor function in a child with cerebral palsy: a case study. J Altern Complement Med. 2010; 16(9):1003-1006. https://doi.org/10.1089/acm.2010.0043

3. Adamu L, Noraniza MA, Rasedee A, Bashir A. Effect of Age and Performance on Physical, Hematological, and Biochemical Parameters in Endurance Horses. J Equine Vet Sci. 2013; 33(6):415-420. https://doi.org/10.1016/j. jevs.2012.07.015

4. Aytekin I, Onmaz AC, Aypak SU, Gunes $V$, Kucuk $O$. Changes in Serum Mineral Concentrations, Biochemical and Hematological Parameters in Horses with Pica. Biol Trace Elem Res. 2011; 139(3):301307. https://doi.org/10.1007/s12011-0108660-y

5. Abeni F, Dal Prà A, Bertin G, Calamari L. Serum protein fraction in mature horses and relationship with metabolic and hematological parameters. J Equine Vet Sci. 2013; 33(11):905-911. https://doi. org/10.1016/j.jevs.2013.01.006

6. Zobba R, Ardu M, Niccolini S, Cubeddu F, Dimauro C, Bonelli $P$, et al. Physical, Hematological, and biochemical responses to acute intense exercise in polo horses. J Equine Vet Sci. 2011; 31(9):542-548. https://doi.org/10.1016/j.jevs.2011.03.010

7. Di Filippo PA, Martins LP, Meireles MAD, de Lannes ST, Peçanha RMS, Graça FAS. Gender differences-induced changes in serum hematologic and biochemical variables in mangalarga marchador horses after a marcha gait competition. J equine Vet Sci. 2016; 43:18-22. https://doi.org/10.1016/j. jevs.2016.04.093

8. Padilha FGF, Dimache LAG, Almeida FQ de, Ferreira AMR, Padilha FGF, Dimache LAG, et al. Blood biochemical parameters of Brazilian sport horses under training in tropical climate. R Bras Zootec. 2017; 46(8):678-682. https://doi.org/10.1590/ s1806-92902017000800008
9. Muñoz A, Riber C, Trigo P, Castejón F. Hematology and clinical pathology data in chronically starved horses. J Equine Vet Sci. 2010; 30(10):581-589. https://doi. org/10.1016/j.jevs.2010.09.002

10. Nikvand AA, Jalali SM, Mashhadi AG, Jalali MR, Amirabadi SH. Clinical, hematologic, hemostatic, and serum biochemical findings related to survival in Arabian horses with colic. Vet C Pathology. 2019; 48(3):441448. https://doi.org/10.1111/vcp.12779

11. Fernández A, Conde T, Fondevila J. La exploración clínica del caballo. Primera. Servet; 2012. http://libros-medicinaveterinaria.blogspot.com/2017/04/laexploracion-clinica-del-caballo-pdf.html

12. Duncan JR, Prasse KW. Patología Clínica Veterinaria. 4 ed. Barcelona, España: Multimedica; 2005. https://www.worldcat. org/title/duncan-prasses-patologia-clinicaveterinaria/oclc/370743899

13. Garcia-Navarro C, Pachaly J. Manual de Hematología veterinaria. São Paulo, Brasil: Varela; 1994. https://www.worldcat.org/ title/manual-de-hematologia-veterinaria/ oclc/46755075

14. Suiza Vet. Manual Veterinario de hematología y coagulación. Lima, Perú: Suiza Vet; 2014. http://www.suizavet.com/manuales/

15. Díaz H, Gavidia C, Li O, Tió A. Valores hematológicos, bilirrubinemia y actividad enzimática sérica en caballos peruanos de paso del valle de Lurín, Lima. Rev Inv Vet Perú. 2011; 22(3):213-222. https://doi. org/10.15381/rivep.v22i3.259

16. Lacerda L, Campos R, Sperb M, Soares E, Barbosa P, Godinho E, et al. Parâmetros hematológicos e bioquímicos em três raças de cavalos de alta performance do Sul do Brasil. Arch Vet Sci. 2006; 11(2):40-44. http://dx.doi.org/10.5380/avs.v11i2.6783

17. Sample SH, Fox KM, Wunn D, Roth E, Friedrichs KR. Hematologic and biochemical reference intervals for adult Friesian horses from North America. Vet C Pathology. 2015; 44(2):194-199. https://doi.org/10.1111/ vcp. 12248 
18. Ono $T$, Yamada $Y$, Hata $A$, Shimokawa Miyama T, Shibano K, Iwata E, et al. Reference values of hematological and blood biochemical parameters for the Noma horse. J Equine Sci. 2019; 30(3):69-73. https:// doi.org/10.1294/jes.30.69

19. Saastamoinen M, Särkijärvi S, Hyyppä S. Garlic (Allium Sativum) Supplementation Improves Respiratory Health but Has Increased Risk of Lower Hematologic Values in Horses. Animals. 2019, 9(1):13-23. https://doi.org/10.3390/ani9010013

20. Kang O-D, Park Y-S. Effect of age on heart rate, blood lactate concentration, packed cell volume and hemoglobin to exercise in Jeju crossbreed horses. J Anim Sci Technol. 2017, 59(2):27-33. https://doi.org/10.1186 Ls40781-017-0126-8

21. Chikhaoui M, Smail F, Adda F. Blood hematological values of Barb horses in Algeria. Open Vet J. 2018; 8(3):330-334. https://doi.org/10.4314/ovj.v8i3.13

22. Čebulj-Kadunc N, Božič $M$, Kosec $M$, Cestnik V. The Influence of Age and Gender on Haematological Parameters in Lipizzan Horses. J Vet Med A Physiol Pathol Clin Med. 2002; 49(4):217-221. https://doi. org/10.1046/j.1439-0442.2002.00439.x

23. Ribeiro CR, Fagliari JJ, Galera PD, Oliveira AR. Hematological profile of healthy Pantaneiro horses. Arq Bras Med Vet Zootec. 2008; 60(2):492-495. https://doi.org/10.1590/ $\underline{\text { S0102-09352008000200033 }}$

24. Shawaf T, El-Deeb W, Hussen J, Hendi M, AlBulushi S. Evaluation of wet cupping therapy on the arterial and venous blood parameters in healthy Arabian horses. Vet World.2018; 11(5):620-626. https://doi.org/10.14202/ vetworld.2018.620-626

25. Aoki T, Ishii M. Hematological and Biochemical Profiles in Peripartum Mares and Neonatal Foals (Heavy Draft Horse). J Equine Vet Sci. 2012; 32(3):170-176. https://doi. org/10.1016/j.jevs.2011.08.015
26. Mariella J, Pirrone A, Gentilini F, Castagnetti C. Hematologic and biochemical profiles in Standardbred mares during peripartum. Theriogenology. 2014; 81(4):526-534. https://doi.org/10.1016/j. theriogenology.2013.11.001

27. Faramarzi B, Rich LJ, Wu J. Hematological and serum biochemical profile values in pregnant and non-pregnant mares. Can J Vet Res. 2018; 82(4):287-293. https://www.ncbi.nlm.nih.gov/pmc/articles/ PMC6168017/

28. AOKI T, KIMURA Y, OYA A, CHIBA A, ISHII $M$, NAMBO Y. Hematological and biochemical features of postpartum fever in the heavy draft mare. J Equine Sci. 2016; 27(1):13-16. https://doi.org/10.1016/j.jevs.2011.08.015

29. Souza AF, Signor J, Schade J, Saito ME, Muller TR, Fonteque JH. Seasonal variation in haematological parameters in crossbred horses used for urban traction from Lages, SC, Brazil. Arch Vet Sci. 2018; 23(3):56-62. http://dx.doi.org/10.5380/avs.v23i3.51021

30. Bis-Wencel $H$, Lutnicki K, Rowicka AZ, Bryl $M$. Long-term exercise and its effect on selected hematological parameters of blood in horses. Med Wet. 2011; 67(6):418-421. https://doi.org/10.5194/aab-62-205-2019

31. Casella S, Vazzana I, Giudice E, Fazio F, Piccione G. Relationship between serum cortisol levels and some physiological parameters following reining training session in horse. An Sci J. 2016; 87(5):729735. https://doi.org/10.1111/asj.12478

32. Larsson J, Pilborg $P H$, Johansen $M$, Christophersen MT, Holte A, Roepstorff L, et al. Physiological Parameters of Endurance Horses Pre- Compared to Post-Race, Correlated with Performance: A Two Race Study from Scandinavia. ISRN Vet Sci. 2013; 2013:684353. https://doi. org/10.1155/2013/684353 